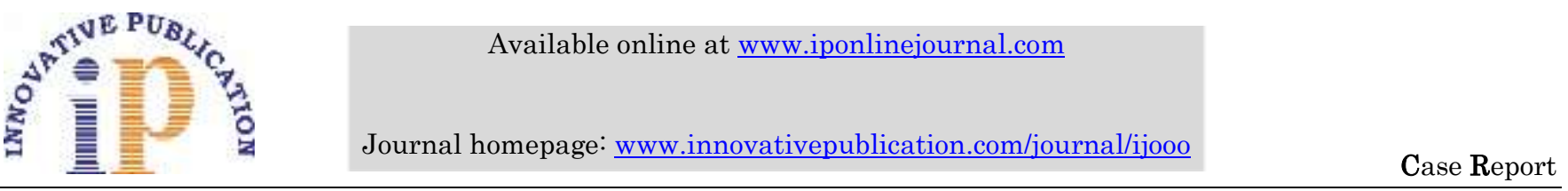

\title{
Unilateral coexisting lymphovenous malformation with persistent fetal vasculature: A case report and short review
}

\author{
Krishna Rao A ${ }^{1}$, Divya Handa ${ }^{2}$, Akshay Sehgal ${ }^{3 *}$, Sindhura Devi $\mathrm{H}^{4}$ \\ ${ }^{1}$ Professor and HOD, ${ }^{2-4}$ Resident, ${ }^{1-4}$ Dept. of Ophthalmology, ${ }^{1-4}$ Kasturba Medical College, Manipal, Karnataka, India
}

\begin{abstract}
Lymphatic malformations are relatively uncommon orbital lesions which are usually diagnosed in early childhood. Their exact etiology is unknown. Persistent Hyperplastic Primary Vitreous (PHPV) results from the failure of the embryological primary vitreous and hyaloid vasculature to regress. We report a rare case of a 9-year old male child who presented to our clinic with unilateral coexisting superficial orbital lymphatic malformation with persistent fetal vasculature manifesting as visual compromise and leukocoria.
\end{abstract}

Keywords: Persistent fetal vasculature, Microphthalmia, Cataract, Lymphangioma, Leukocoria.

\section{Introduction}

Lymphatic malformations, also referred as lymphangiomas, are uncommon benign venolymphatic lesions which are characterized by dilation of lymphatic vessels which contain lymph. Lymphatic malformations occurring in the orbit are relatively uncommon and are usually diagnosed in early childhood. The location in the orbit can be superficial (no extension posterior to the equator of the globe), deep (exclusively posterior to the equator) or combined (having both superficial and deep components). Deep orbital malformations often remain unrecognized and the superficial lesions are detected and diagnosed much sooner and are generally more of a cosmetic concern. Lymphatic malformations are less likely to cause visual compromise if present superficially and alone. ${ }^{1,2}$

Persistent hyperplastic primary vitreous (PHPV) is characterized by the persistence of various portions of the primary vitreous with hyperplasia of the associated embryonic connective tissue. It presents as leukocoria and is commonly associated with visual compromise due to the associated ocular morbidities like microphthalmia, cataract and glaucoma. ${ }^{3}$

\section{Case Report}

A 9-year old male child was brought to the ophthalmology out-patient clinic by his parents and he complained of diminution of vision and grittiness sensation in his right eye. His parents informed us about the presence of a visible reddish mass in the right eye since birth. On ocular examination, his best corrected visual acuity in the right eye was counting fingers at 1 meter and 6/9 in the left eye. He was found to have a 30-degree esotropia in the right eye which was not taking up fixation. (Fig. 1) Extra-ocular movements were full bilaterally. On examination of the lids and adnexa, there was fullness of the area around the right medial canthus. There was no ptosis or proptosis. Right eye anterior segment examination revealed leukocoria, microphthalmia, a painless reddish vascular bulbar conjunctival mass and a dense posterior subcapsular cataract. The reddish mass was present in the nasal, inferior and temporal aspects of the bulbar conjunctiva with extension to both the canthi, however, its inferior border was not identified. Its superior border extended up to 10 clock hours temporally and 2 clock hours nasally in the perilimbal area. (Fig. 2,3 and 4) Right eye dilated fundus examination revealed persistent fetal vasculature with a retrolental fibrovascular membrane extending from the optic disc and associated optic disc hypoplasia. (Fig. 5) Intraocular pressure measured by goldmann applanation tonometry was $18 \mathrm{~mm} \mathrm{Hg}$ in the right eye and $16 \mathrm{~mm} \mathrm{Hg}$ in the left eye. Examination of left eye was completely normal. Systemic examination was unremarkable. There was no significant medical history.

A contrast enhanced computed tomography (CECT) scan of the orbit was performed which demonstrated microphthalmia of the right eye and findings consistent with a unilateral right-sided superficial orbital lymphovenous malformation involving the nasal, inferior and temporal aspects of the globe, anterior to the equator. The mass was seen to be approximating the right ethmoid sinus and the nasal septum was slightly deviated towards the left side. (Fig. 6 and 7).

Thus, the child was diagnosed to have a right-sided superficial orbital lymphovenous malformation with rightsided esotropia, microphthalmia, posterior subcapsular cataract with persistent fetal vasculature. The child's parents were explained and counselled regarding the conditions and the associated guarded visual prognosis. They were given the option of surgical excision with biopsy of the lymphovenous malformation, but they opted for regular follow-up and close observation. He was prescribed tear supplements in the right eye for symptomatic relief of the grittiness.

*Corresponding Author: Akshay Sehgal, Dept. of Ophthalmology, Kasturba Medical College, Manipal, Karnataka, India

Email: akshaysehgal08@gmail.com

http://doi.org/ 10.18231/j.ijooo.2019.040 


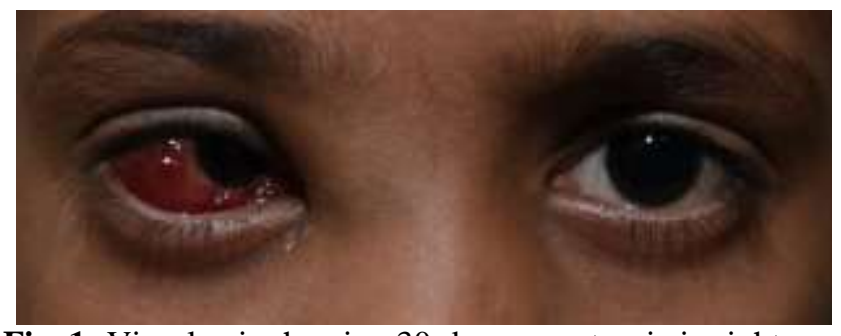

Fig. 1: Visual axis showing 30-degree esotropia in right eye along with microphthalmia and a reddish vascular mass.

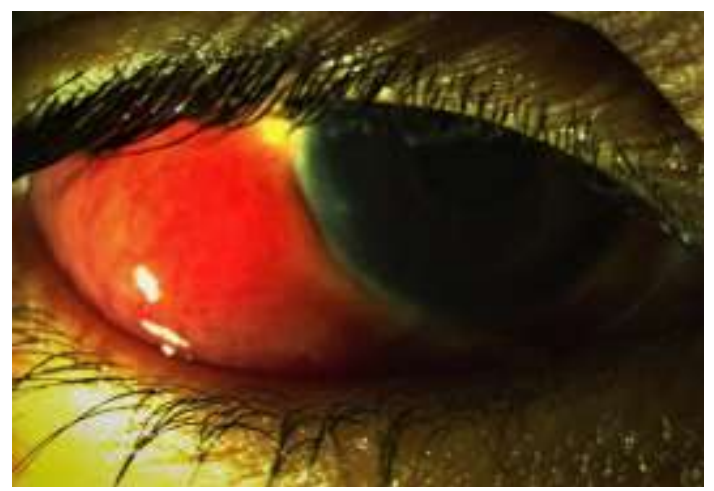

Fig. 2: Reddish vascular bulbar conjunctival mass in the right eye.

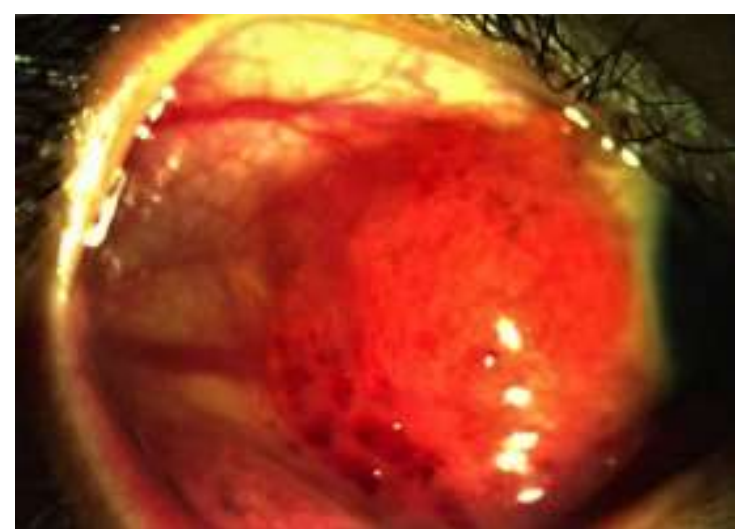

Fig. 3: Temporal extent of the reddish vascular bulbar conjunctival mass in the right eye.

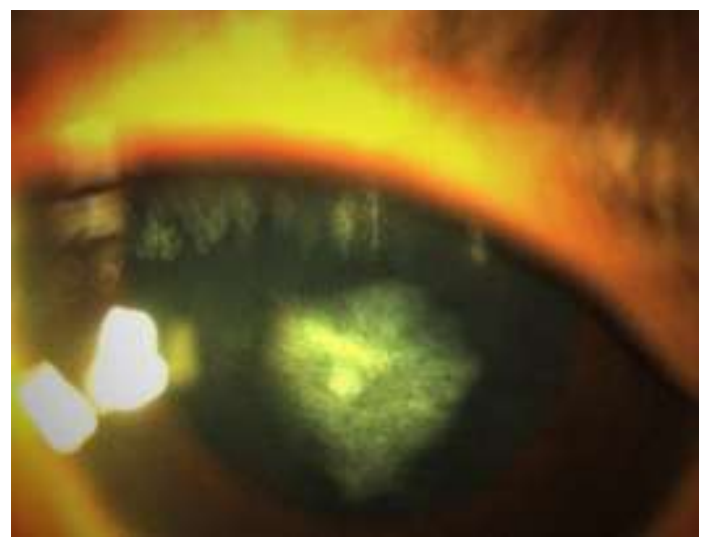

Fig. 4: Slit lamp examination of anterior segment of right eye showing dense posterior subcapsular cataract.

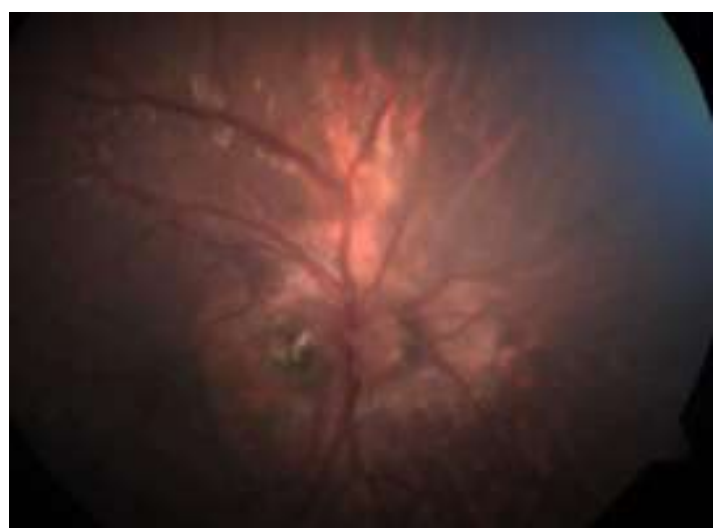

Fig. 5: Fundus examination of right eye showing features of persistent fetal vasculature including optic disc hypoplasia.

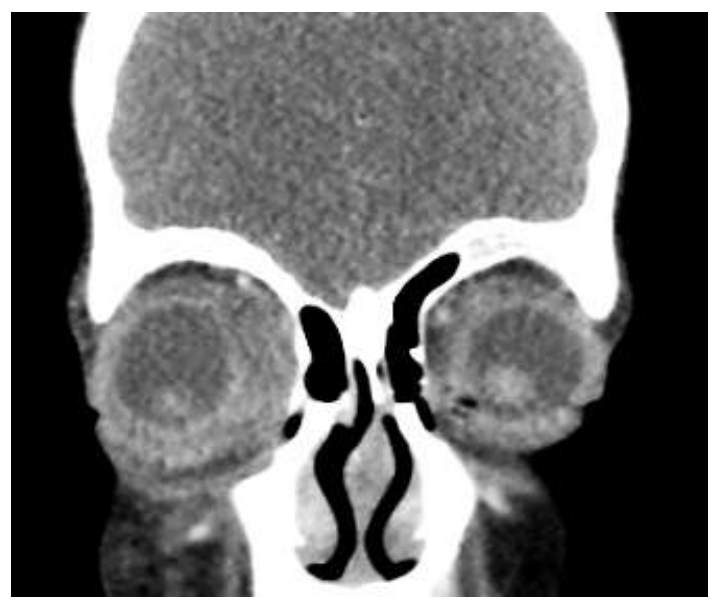

Fig. 6: Contrast enhanced $\mathrm{CT}$ in coronal plane showing right-sided superficial orbital lymphonvenous malformation

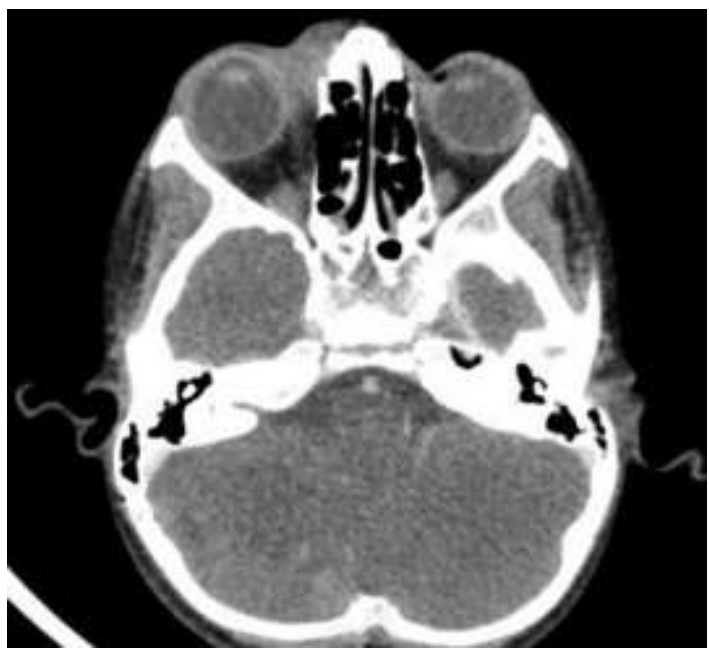

Fig. 7: Contrast enhanced CT in axial plane showing rightsided superficial orbital lymphonvenous malformation

\section{Discussion}

Our literature search revealed that unilateral coexisting persistent fetal vasculature with orbital lymphovenous malformation has not been reported yet. Orbital lymphatic malformations also known as orbital lymphangiomas, are 
benign vascular and lymphatic tumours of the head and neck which are usually diagnosed in early childhood. ${ }^{2,4}$ They represent $1-3 \%$ of the orbital masses. ${ }^{2}$ They can present with ptosis, proptosis, restriction of ocular motility, compressive optic neuropathy and disfigurement. ${ }^{4}$ The exact etiology of a conjunctival lymphatic malformation is not yet understood. It is usually of sporadic occurrence and can be occasionally associated with the Turner syndrome or the Nonne-MilroyMeige disease. It is most commonly present in the bulbar conjunctiva, similar to our patient. ${ }^{1}$ Orbital imaging by computed tomography (CT) and magnetic resonance imaging (MRI) are essential for making the correct diagnosis. Orbital ultrasound and biopsy are also valuable in diagnosing the condition. ${ }^{5}$ Visual prognosis is generally good, especially if they are superficial and present alone. Surgical removal can be considered for their treatment. Other treatment modalities such as percutaneous sclerotherapy have also been tried for their removal and it has been found to be a safe and effective treatment for orbital lymphatic malformations as a primary treatment or for recurrence after surgical intervention. ${ }^{2}$ There is no gold standard for treatment and regular follow-up with observation has been opted for by clinicians and patients in many instances like our patient and his family. ${ }^{4}$

This child had decreased visual acuity due to the coexistence of persistent hyperplastic primary vitreous (PHPV) with the lymphovenous malformation and other associated ocular morbidities like cataract. PHPV is a rare congenital developmental malformation of the eye in which there is a retrolental fibrovascular mass caused by persistence of the primary vitreous that normally regresses during the embryonic development of the eye. The condition can occur in isolation or with other ocular disorders. Most cases are sporadic in occurrence, but it can also be inherited as an autosomal dominant or recessive trait. ${ }^{6,7}$ It is unilateral in $90 \%-98 \%$ of cases. Rare bilateral PHPV is usually associated with neurologic and systemic anomalies, such as Norrie disease and Warburg syndrome. ${ }^{7}$

In the embryo, the primitive lens and the vitreous are supplied by the hyaloid blood system, which mainly includes the hyaloid artery, a branch of the ophthalmic artery, which supplies the central primary vitreous. The peripheral part of the primary vitreous is supplied by the vasa hyaloidea propria whereas the iris and lens are supplied by the anterior ciliary vessels. The anterior portion of the arterial system usually regresses by about 8 months of life and the posterior system regresses by 7 months of life. ${ }^{7}$ Persistence of the fetal vasculature can be classified as anterior type of PHPV which can include a shallow anterior chamber predisposing to glaucoma, cataract and the classic retrolental fibrovascular membrane, and the posterior type of PHPV which may be associated with developmental abnormalities of the optic nerve or surrounding retina which can be scarred or even detached. The differential diagnosis for PHPV includes retinoblastoma, Coats disease, retinopathy of prematurity, optic nerve drusen and toxocariasis as leukocoria may be seen at presentation in all these conditions. ${ }^{7,8}$

There is generally a poor visual outcome in patients with PHPV because of the associated conditions such as glaucoma, retinal detachment and deprivation amblyopia. Other factors contributing towards visual morbidity can include corneal opacity and cataracts. The cataract may range from a tiny visually insignificant opacity to a wide spread vascularized plaque on the back of the lens (like in our patient) and sometimes even a total white cataract. The visual outcome of the posterior type of persistent fetal vasculature is usually poorer compared to the the anterior type. Patients with the pure anterior form may have a good visual outcome by cataract extraction followed by contact lens correction and amblyopia therapy. Usually, patients have a combination of the anterior and posterior types of persistent fetal vasculature, like the findings in our patient who had strabismus, leukocoria, microphthalmia, cataract and optic disc hypoplasia, which collectively led to the poor visual acuity. ${ }^{7,8}$

Thus, persistent fetal vasculature is an important differential diagnosis which should be kept in mind by ophthalmologists while examining a child who has presented with leukocoria, strabismus and low visual acuity. Goals in treatment are saving the eye from the complications of untreated PHPV while evaluating the benefit of a lensectomy with vitrectomy, saving of useful vision and achieving acceptable cosmetic outcome. Timely diagnosis and appropriate management with regular followup may prevent further deterioration of vision in some patients. A meticulous eye examination is essential at presentation, as occasionally, persistent fetal vasculature can present with a coexisting ocular condition such as the lymphatic malformation in our patient.

\section{Source of Funding}

None.

\section{Conflict of Interest}

None.

\section{References}

1. Seca M, Borges P, Reimao P, Gomes M, Meireles A. Conjunctival lymphangioma: a case report and brief review of the literature. Case Rep Ophthalmol Med 2012;2012.

2. Hill III RH, Shiels WE, Foster JA, Czyz CN, Stacey A, Everman KR, Cahill KV. Percutaneous drainage and ablation as first line therapy for macrocystic and microcystic orbital lymphatic malformations. Ophthalmic Plast Reconstr Surg 2012 Mar 1;28(2):119-25.

3. Anteby I, Cohen E, Karshai I, BenEzra D. Unilateral persistent hyperplastic primary vitreous: course and outcome. J Am Assoc Pediatr Ophthalmol Strabismus 2002 Apr 30;6(2):92-9.

4. Patel SR, Rosenberg JB, Barmettler A. Interventions for orbital lymphangioma. Cochrane Database of Syst Rev 2019(5).

5. Khan SN, Sepahdari AR. Orbital masses: CT and MRI of common vascular lesions, benign tumors, and malignancies. Saudi J Ophthalmol 2012;26(4):373-83 
6. Shastry BS. Persistent hyperplastic primary vitreous: congenital malformation of the eye. Clin Exp Ophthalmol 2009;37(9):884-90.

7. Li CD, Meltzer DE. Case 206: persistent hypertrophic primary vitreous. Radiol 2014;271(3):921-5.

8. MacKeen LD, Nischal KK, Lam WC, Levin AV. Highfrequency ultrasonography findings in persistent hyperplastic primary vitreous. J Am Assoc Pediatr Ophthalmol Strabismus 2000;4(4):217-23.

How to cite this article: Rao KA, Handa D, Sehgal A, Devi $\mathrm{SH}$. Unilateral coexisting lymphovenous malformation with persistent fetal vasculature: A case report and short review. Int J Ocul Oncol Oculoplasty 2019;5(3):153-6. 